\title{
Correction to: Restoration of MARCKS enhances chemosensitivity in cancer
}

\author{
Tim Wenzel ${ }^{1} \cdot$ Thomas Büch $^{2} \cdot$ Nicole Urban $^{1} \cdot$ Ulrike Weirauch $^{2} \cdot$ Katrin Schierle $^{3} \cdot$ Achim Aigner $^{2}$. \\ Michael Schaefer ${ }^{1} \cdot$ Hermann Kalwa ${ }^{1}$
}

Published online: 28 February 2020

(c) Springer-Verlag GmbH Germany, part of Springer Nature 2020

\section{Correction to: Journal of Cancer Research and Clinical Oncology https://doi.org/10.1007/s00432-020-03149-2}

In the original article, the title of the article is "Restoration of MARCK enhances chemosensitivity in cancer". The authors would like to change the article title to "Restoration of MARCKS enhances chemosensitivity in cancer" by adding a letter "S" to the word MARCK.

Publisher's Note Springer Nature remains neutral with regard to jurisdictional claims in published maps and institutional affiliations.
The original article can be found online at https://doi.org/10.1007/ s00432-020-03149-2.
Michael Schaefer

michael.schaefer@medizin.uni-leipzig.de

1 Rudolf-Boehm-Institut für Pharmakologie und Toxikologie, Medizinische Fakultät, Universität Leipzig, Härtelstraße 16-18, 04107 Leipzig, Germany

2 Selbständige Abteilung für Klinische Pharmakologie Rudolf-Boehm-Institut für Pharmakologie und Toxikologie, Medizinische Fakultät, Universität Leipzig, Härtelstraße 16-18, 04107 Leipzig, Germany

3 Institut für Pathologie, Medizinische Fakultät, Universität Leipzig, Liebigstraße 26, 04103 Leipzig, Germany 